Ricardo Luiz Lorenzi ${ }^{1}$

Ivone Martini de Oliveira²

\section{Tuberculose em trabalhadores de Enfermagem: uma abordagem epidemiológica de base populacional}

\author{
Tuberculosis in nursing personnel - a population based \\ epidemiological approach
}

${ }^{1}$ Coordenação de Vigilância em Saúde de São Paulo (Covisa-SMS-SP). Subgerência de Vigilância em Saúde do Trabalhador.

${ }^{2}$ Conselho Regional de Enfermagem de São Paulo (Coren-SP) e Associação Nacional de Enfermagem do Trabalho (Anent).

Contato:

Ricardo Luiz Lorenzi

Rua Santa Isabel, 181 - Vila Buarque - São Paulo

CEP: 01221-010

E-mail:

rlorenzi@usp.br
Recebido: 31/08/2007

Revisado: 21/07/2008

Aprovado: 11/08/2008

\section{Resumo}

Introdução: O risco de tuberculose em trabalhadores de saúde tem suscitado crescente interesse na sociedade e entre pesquisadores dos países desenvolvidos. No Brasil, porém, persistem lacunas de conhecimento e de ação prática sobre os aspectos epidemiológicos do problema. Este deve ser investigado em detalhe, para que melhor se embasem e apliquem as normas e regulamentos sanitários e de segurança, como é o caso da NR-32. Métodos: Com base em dados do EPI-TB (banco de dados do Programa de Controle de Tuberculose de São Paulo), os autores constroem estimativas de risco para o coletivo de enfermagem, para 2004, usando a razão de incidências padronizada e tendo a população paulistana como referência. Descrevem o tipo de estabelecimento empregador com base em dados do Conselho Regional de Enfermagem de São Paulo - Coren-SP. Resultados: Aproximadamente 90\% dos casos registrados pelo instrumento de notificação do EPI-TB tiveram sua ocupação confirmada no Coren-SP. A faixa de 20 a 29 anos apresentou um risco maior de tuberculose-doença entre enfermeiros que o da população de referência. Entre auxiliares de enfermagem, observou-se risco aumentado na faixa de 15 a 19 anos. Conclusões: $\mathrm{O}$ coletivo de enfermagem pode ser tomado como categoria-índice da tuberculose nosocomial e o monitoramento desta considerado exeqüível desde que sejam introduzidas modificações no instrumento de notificação. São abordadas possíveis implicações de ações articuladas entre diferentes atores institucionais na prevenção e controle do agravo, discutindo-se a necessidade de superação de práticas anacrônicas de Vigilância em Saúde.

Palavras-chaves: tuberculose / epidemiologia, vigilância em saúde do trabalhador, vigilância sanitária de serviços de saúde, enfermagem.

\begin{abstract}
Introduction: The risk of tuberculosis in health-care workers has brought growing concerns to society and researchers from developed countries. In Brazil there is still a gap between epidemiological knowledge and know-how in this field. This problem requires a more detailed investigation to give support and to provide better application to occupational safety and health regulations, such as the Regulatory Act called "NR-32". Methods:Using Sao Paulo Tuberculosis Program database "EPI-TB" and calculating the Standardized Incidence Ratio, the authors estimated nursing personnel risks. The reference for the study was the population in the city of Sao Paulo in the year 2004. Employers' profiles are described according to health-care facility status, based upon the state of Sao Paulo Nursing Council - Coren's database. Results: Approximately 90\% of cases registered by the EPI-TB notification instrument had their occupation validated by Coren's database. The 20-29 age interval showed an increased risk among nurses when compared to the reference population. Among 15 to19 year-old nurses'aides there was also an increase of risk. Conclusions: Nursing personnel may be considered as a nosocomial tuberculosis index category, and monitoring nosocomial TB seems to be feasible since changes are provided in the notification instrument of this disease. The study has tackled possible implications of articulated actions between different institutional actors on prevention and control of tuberculosis, and it discusses ways to overcome current anachronisms in Health Surveillance practices.
\end{abstract}

Keywords: tuberculosis / epidemiology, surveillance of the workers health, health surveillance of health services, nursery. 


\section{Introdução}

A informação quantitativa sobre riscos ocupacionais na América Latina é escassa e possui sérias limitações. Mesmo quando os trabalhadores estão cobertos por um sistema de notificação, a identificação precária das doenças ocupacionais e os aspectos burocráticos desses sistemas levantam questões sobre sua validade e acurácia (BUVINIC; GIUFFRIDA; GLASSMAN, 2003).

No Brasil, até recentemente, apenas estatísticas de $\mathrm{CAT}^{3}$ serviam de base para construção de indicadores e planejamento, com a séria limitação de incluir basicamente apenas os acidentes de trabalho (e alguns outros agravos) em trabalhadores formais. Com a recente instituição da notificação compulsória de agravos à saúde do trabalhador no Sinan ${ }^{4}$, ampliou-se a cobertura destes para além dos acidentes e do setor formal da economia. Entretanto, o sistema ainda cobre um elenco limitado dos agravos que podem ser relacionados ao trabalho e se ressente da dificuldade que os serviços demonstram em identificar essa relação e notificá-la. Nesse contexto, emerge uma questão: o que interessa à saúde do trabalhador que possa escapar aos sistemas CAT e Sinan?

A tuberculose (TB) se constitui num sério problema de saúde no Brasil, bem como em outros países em desenvolvimento, e pode ser considerada doença relacionada ao trabalho para o segmento de trabalhadores em atividades que propiciam contato direto com produtos contaminados ou com doentes bacilíferos (BRASIL. Ministério da Saúde; ORGANIZAÇÃO PANAMERICANA DA SAÚDE, 2001). Há dificuldade em se determinar o risco ocupacional relacionado com a tuberculose em países em desenvolvimento por falta de dados sobre a incidência da doença em profissionais de saúde, existência de elevada prevalência de infecção tuberculosa e doença na população geral, uso disseminado da vacina com o Bacilo Calmétte-Guerin (BCG) e dificuldade de obtenção de dados sobre a prevalência de infecção pelo HIV em profissionais de saúde. Há poucos trabalhos investigando essa associação no Brasil, em sua maior parte, estudos acadêmicos (FRANCO; ZANETTA, 2004). Outrossim, as doenças respiratórias relacionadas ao trabalho não estão suficientemente contempladas nas bases de dados construídas com propósitos de vigilância epidemiológica (BAGATIN et al., 2006).

A incidência de tuberculose entre trabalhadores de saúde, em particular os de enfermagem, tem sido objeto de muitos estudos nos países industrializados devido às exposições múltiplas a que estão sujeitos esses profissionais ao bacilo de Koch e à emergência de formas resistentes da doença às drogas (KRÜÜNER et al., 2001). Diferentemente, porém, de problemas de saúde do trabalhador, tais como exposição a agentes químicos ou poeiras, a verossimilhança da exposição ocupacional à tuberculose possui uma íntima relação com o risco de tuberculose na comunidade circunvizinha ao Estabelecimento de Assistência à Saúde (EAS). Esse aspecto torna de particular importância o conhecimento dos perfis epidemiológicos da doença na comunidade e nos trabalhadores do setor e das relações entre ambos para a sua prevenção e controle. Ou seja, para a efetivação plena de normas e regulamentos sanitários, concorrem, também, o conhecimento da estrutura epidemiológica das doenças relacionadas e da dinâmica daquelas relações.

No Município de São Paulo, iniciou-se um processo de discussão sobre os aspectos ocupacionais que envolvem a TB e a necessidade de se avaliar a exposição ocupacional e a adequação dos ambientes de trabalho em serviços de saúde, sob a vigência da Norma Regulamentadora NR-32 (BRASIL, 2005). Assim, em 2005 foi realizado um levantamento exploratório da incidência de TB entre trabalhadores da saúde, sobre o banco de dados EPI-TB (LORENZI; ANDRADESILVA, 2005). Este mostrou em números absolutos a participação expressiva das categorias de enfermagem no conjunto de trabalhadores da saúde notificados. A partir de então, buscaram-se os elementos necessários para essa análise descritiva. A aproximação entre as instituições envolvidas (Anent, Covisa, Coren e CVECentro de Vigilância Epidemiológica do Estado de São Paulo) foi buscada com base nas competências das mesmas, no pressuposto de complementaridade das ações em diferentes níveis de atuação no Sistema Único de Saúde e na cooperação técnica que as mesmas podiam prover ao projeto.

Os objetivos deste trabalho foram: apresentar a incidência de tuberculose (todas as formas) entre trabalhadores de enfermagem do município de São Paulo, comparando-a com a da população geral no ano de 2004; estimar seu risco de TB-doença; descrever o indivíduo notificado segundo o local de trabalho (tipo de EAS onde trabalha); discutir as implicações e os possíveis desdobramentos de ações articuladas de vigilância na vigência da NR-32, frente aos aspectos epidemiológicos desse agravo.

\section{Material e métodos}

\section{Fonte de dados}

a) EPI-TB (CVE-SP) - banco de dados dos pacientes residentes na cidade de São Paulo;

\footnotetext{
${ }^{3}$ Comunicação de Acidentes do Trabalho.

${ }^{4}$ Sistema Nacional de Agravos de Notificação.
} 
b) Cadastro de profissionais inscritos no Coren-SP.

\section{Critérios de inclusão}

Foram considerados registros válidos de profissionais com inscrições vigentes no Coren-SP até 2004 e residentes na capital. Os denominadores populacionais atenderam à mesma lógica.

\section{Construçấo dos coeficientes}

Os da população geral foram calculados sobre estimativa populacional do IBGE. Para o cálculo dos coeficientes da população de enfermagem, foram selecionados os casos novos referentes a todas as formas clínicas de tuberculose incidentes no ano de 2004. Esses casos de interesse foram identificados no EPI-TB por meio de recorte dos campos "tipo de ocupação" e "ocupação" e confirmados no Coren, que também forneceu os denominadores da população sob risco, em 2004, por faixa etária. Cada trabalhador cadastrado foi considerado como exposto pelo período de um ano. Dado o pequeno número de atendentes e técnicos, estes foram agrupados em conjunto com os auxiliares.

Os coeficientes por grupo etário da população de referência foram comparados graficamente com os da população de estudo. As curvas de incidência foram suavizadas no $\mathrm{Excel}^{\circledR}$. Admitiu-se a incidência de TB nesses trabalhadores como variável operacional (proxy) da incidência ocupacional (nosocomial) na população estudada. Os valores de referência figuram na Tabela 1.

\section{Padronização dos coeficientes}

Feita através de técnica indireta, calculando-se a Razão de Incidências Padronizada (Standardized Incidence Ratio) por categoria profissional (enfermeiro, técnico e auxiliar), tomando a população do município como a de referência. Construiu-se o intervalo de confiança de $95 \%$ para a $S I R$, para cada categoria, segundo a distribuição de Poisson.

\section{Tipos de EAS's empregadores}

Foram construídas quatro categorias de vínculos: só público, assinalado quando o trabalhador possuía um ou mais vínculos, mas todos públicos; só privado, quando possuía um ou mais vínculos, mas todos privados; só universitário, quando só era informado vínculo com instituição universitária; e de múltiplo tipo, quando havia mescla entre os tipos. A identificação dos empregadores também foi obtida no Coren, que registra essa informação referida e atualizada pelos próprios profissionais inscritos. Não foi possível investigar os vínculos empregatícios de seis casos no banco do Coren, levantados no Epi-TB a posteriore.

\section{Aspectos éticos}

A identificação dos trabalhadores no Coren obedeceu a preceitos éticos, com a identificação do indivíduo reservada ao propósito de levantamento epidemiológico e nenhum contato foi estabelecido entre os pesquisadores e os casos.

\section{Resultados}

Os casos confirmados (quanto à ocupação) por busca no cadastro do Coren figuram na Tabela 2.

Os coeficientes de incidência, por 100.000 hab., foram: 114 para enfermeiros; 65 para técnicos; 75 para auxiliares; 61 para a população geral. As Razões de Incidências Padronizadas figuram nas Tabelas 3 e 4.

Tabela 1 Coeficientes de incidência de tuberculose (todas as formas, ambos os sexos), por 100 mil habitantes. Município de São Paulo, 2004

\begin{tabular}{cccc}
\hline Faixa etária & $n^{0}$ casos & População & Coef. incid. \\
\hline 00 a 04 & 102 & 916.420 & 11,1 \\
05 a 09 & 73 & 861.559 & 8,5 \\
10 a 14 & 93 & 921.254 & 10,1 \\
15 a 19 & 399 & 1.030 .410 & 38,7 \\
20 a 29 & 1649 & 2.041 .313 & 80,8 \\
30 a 39 & 1539 & 1.761 .226 & 87,4 \\
40 a 49 & 1271 & 1.397 .106 & 91,0 \\
50 a 59 & 780 & 880.791 & 88,6 \\
60 ou + & 632 & 994.787 & 63,5 \\
\hline Total * & 6572 & 10.804 .867 & 60,8 \\
\hline
\end{tabular}

Fonte: SMS-SP- Covisa /CCD (EpiTb - residentes)

* Calculado com o acréscimo de 34 indivíduos de idades ignoradas. 
Tabela 2 Índice de confirmação ocupacional dos casos de TB notificados (todas as formas, ambos os sexos) no cadastro do Coren-SP. Município de São Paulo, 2004

\begin{tabular}{lccc}
\hline \multicolumn{1}{c}{ Categoria } & Registrados no Epi-TB & Confirmados no Coren-SP & Confirmação ocupacional (\%) \\
\hline Enfermeiros & 14 & $14\left(15^{*}\right)$ & 100,0 \\
Técnicos & 4 & $1\left(3^{* * *}\right)$ & 25,0 \\
Auxiliares & 44 & 38 & 86,4 \\
Atendentes & 3 & 1 & 33,3 \\
\hline Total & 65 & $54\left(57^{* * * * * *}\right)$ & $83,1\left(87,7^{* * * * * * * *}\right)$ \\
\hline
\end{tabular}

*Acrescidos de um registro de técnico de enfermagem verificado como de enfermeiro.

*** Acrescidos de dois registros de auxiliar verificados como de técnicos.

****** Incluídos registros transpostos de categorias.

Tabela 3 Razão de incidências padronizadas (SIR) de TB (todas as formas, ambos os sexos) em auxiliares de enfermagem. Município de São Paulo, 2004

\begin{tabular}{cccccc}
\hline Idade & Observado (O) & Esperado (E) & $\begin{array}{c}\text { SIR\% } \\
(\text { O/E) } 100\end{array}$ & \multicolumn{2}{c}{$\begin{array}{c}\text { SIR\% } \\
\text { [IC 95\%] }\end{array}$} \\
\hline 15 a 19 & 1 & 0.0012 & $86082.7^{*}$ & 2179 & 479621 \\
20 a 29 & 6 & 5.8615 & 102.4 & 38 & 223 \\
30 a 39 & 11 & 13.4429 & 81.8 & 41 & 146 \\
40 a 49 & 10 & 13.6033 & 73.5 & 35 & 135 \\
50 a 59 & 8 & 9.1302 & 87.6 & 38 & 173 \\
60 ou + & 3 & 2.5825 & 116.2 & 24 & 339 \\
\hline Total & 39 & 44.6216 & 87.4 & 62 & 119 \\
\hline " $<<0,01$ & & & &
\end{tabular}

Tabela 4 Razão de incidências padronizadas (SIR) de TB (todas as formas, ambos os sexos) em enfermeira(o)s. Município de São Paulo, 2004

\begin{tabular}{cccccc}
\hline Idade & Observado(O) & Esperado (E) & $\begin{array}{c}\text { SIR\% } \\
(\text { O/E) } \times 100\end{array}$ & \multicolumn{2}{c}{$\begin{array}{c}\text { SIR\% } \\
\text { [IC 95\%] }\end{array}$} \\
\hline 20 a 29 & 5 & 1.3070 & $382.5^{*}$ & 124 & 893 \\
30 a 39 & 4 & 3.3773 & 118.4 & 32 & 303 \\
40 a 49 & 5 & 4.3677 & 114.5 & 37 & 267 \\
50 a 59 & 1 & 1.9075 & 52.4 & 1 & 292 \\
60 ou + & 0 & 0.4892 & 0.0 & 0 & $754+$ \\
\hline Total & 15 & 11.4487 & 131.0 & 73 & 216 \\
\hline " $<<0,05$ & & & & &
\end{tabular}


A faixa etária de maior incidência bruta para enfermeiros foi a de 20 a 29 anos e para auxiliares as maiores incidências foram sobre as faixas de 30 a 39 e de 40 a 49 anos (Gráfico 1).

Quanto aos tipos de EAS empregadores, para $25 \%$ dos casos havia informação de vínculo de trabalho em serviços de saúde universitários (Gráfico 2).

No Gráfico 3, estão comparados os coeficientes de incidência da população em geral e de enfermeiras, por grupo etário, em intervalos decenais.

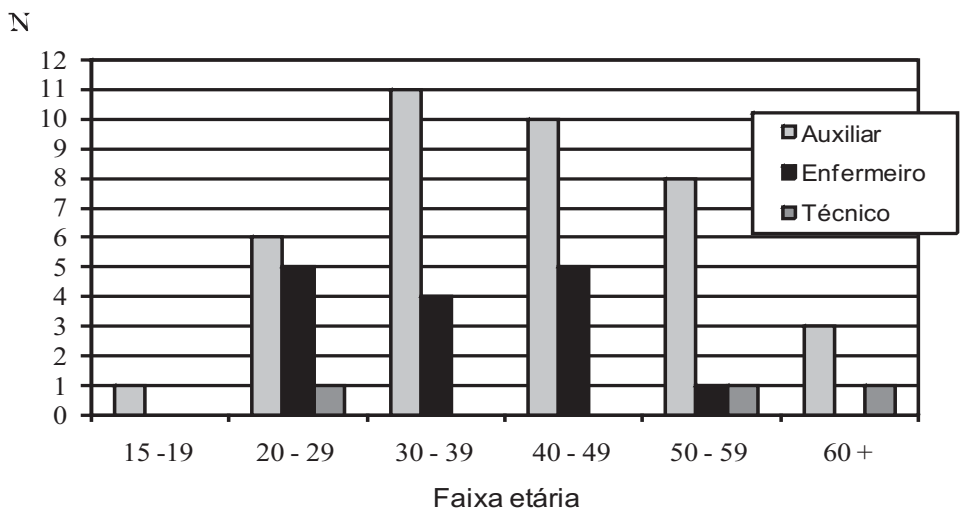

Gráfico 1 Distribuição etária dos casos incidentes de Tb em profissionais de enfermagem, segundo categoria de inscrição no Coren, São Paulo, 2004

$\mathrm{N}$

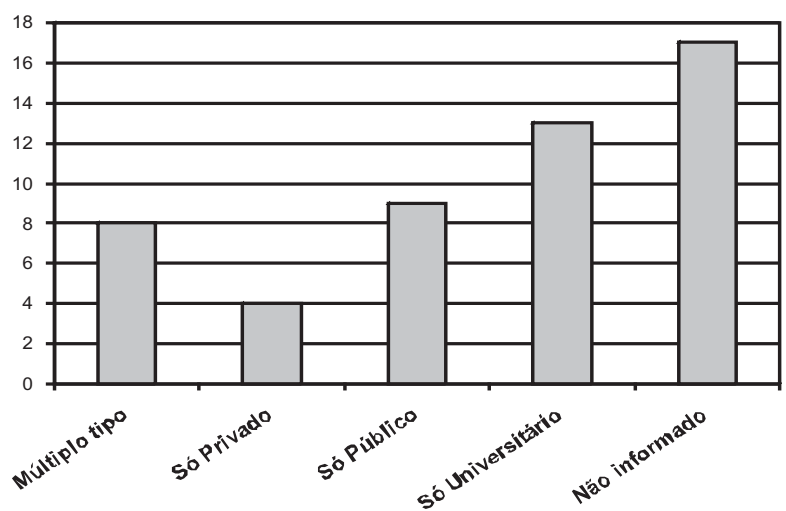

Gráfico 2 Tipo de estabelecimento de Assistência à Saúde empregador informado ao Coren-SP

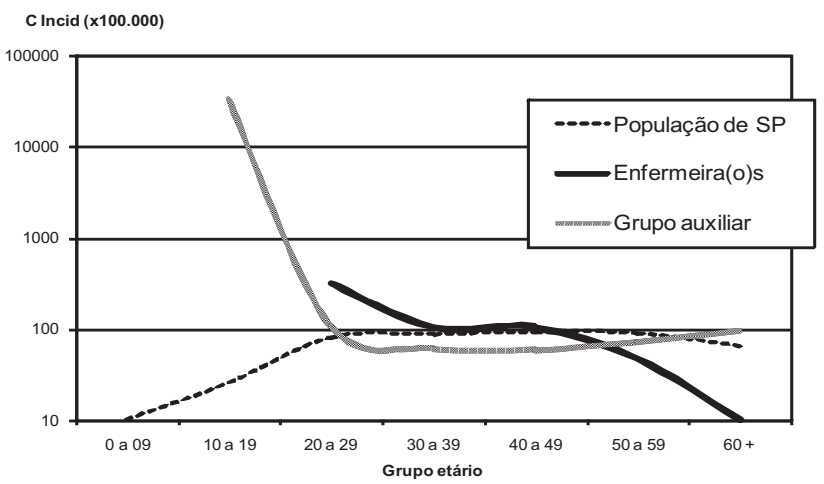

Gráfico 3 Coeficientes de incidência de Tb específicos por grupo etário. Município de São Paulo, 2004 


\section{Discussão}

Na Tabela 2, observa-se um índice elevado de confirmação ocupacional dos casos, revelando boa especificidade dessa informação no EPI-TB, na população estudada. Esse aspecto pode estar relacionado com o perfil de formação dos técnicos envolvidos em ações de vigilância epidemiológica no município, muitos dos quais da área de enfermagem. Por essa lógica, os casos notificados de pessoal de enfermagem tenderiam a ser mais bem registrados e investigados. Mas, considerando o estigma que a doença ainda carrega, é oportuno indagar se o diagnóstico e o fato de ser trabalhador da saúde não inibem a declaração de ocupação pelo doente no momento da notificação. Nesse caso, ocorreria alguma perda de sensibilidade para essa informação, equivalendo a uma "subnotificação" no campo ocupacional.

Analisando a distribuição dos casos por tipo de empregador, verificam-se vários tipos de arranjos: público+privado, privado+universitário etc. Ressalte-se que, dos casos de vínculo informado, 38\% se referem a trabalhadores que atuam em hospitais universitários, o que leva a supor que nesses espaços o problema da TB ocupacional é no mínimo de dimensões semelhantes aos de outros EAS. É preciso, porém, olhar com cautela para a alta proporção de indivíduos que não informaram seus vínculos (33\%); estes poderiam, em tese, alterar o perfil.

Um maior número de vínculos empregatícios está associado a uma tendência de deterioração da remuneração; verifica-se, sobre a base da $\mathrm{RAIS}^{5}$, que $32 \%$ dos enfermeiros, $26 \%$ dos auxiliares e $23 \%$ dos atendentes de enfermagem possuíam dois ou mais vínculos em 2001, no Brasil (DEDECCA et al., 2004). Em acréscimo, esses trabalhadores têm um perfil predominantemente feminino, o que os expõe, em sua maioria, a uma tripla jornada de trabalho (uma constituída pelo trabalho no lar). A participação feminina nesse mercado formal era, em 1995, de 88,2\% entre os enfermeiros, $86,5 \%$ entre os atendentes de enfermagem e $86,1 \%$ entre os auxiliares (GIRARDI; CARVALHO, 2003). Logo, para um grande contingente desses trabalhadores, longos períodos de trabalho são uma constante, agravada por longas distâncias entre domicílios e locais de trabalho que muitos destes enfrentam. Nesse cenário, é lícito admitir que aumente sua suscetibilidade frente ao desgaste bio-psíquico envolvido no trabalho, no deslocamento (trajeto) e na exposição à crescente precarização das relações de trabalho.

Identificou-se um excesso significativo de casos de TB no grupo etário mais jovem de enfermeiras e de auxiliares comparado com a população geral. Esse achado alerta sobre a necessidade de medidas mais efetivas de controle da doença no início da vida pro- fissional. O perfil etário da incidência se assemelha ao encontrado por Raitio, Henenius e Tala (2003) em trabalhadores de enfermagem finlandeses: a faixa de 20 a 39 anos apresenta uma magnitude nitidamente superior à de 40 a 59 anos. O risco que esses trabalhadores mais jovens experimentam pode estar relacionado mais a variáveis comportamentais (permanecer mais tempo em contato com o paciente, por exemplo) do que a um componente biológico. Uma exposição aumentada no início da atividade profissional é esperada em razão de cargas de trabalho maiores e de inexperiência. A implicação sobre a saúde do trabalhador é evidente: aspectos da formação desses profissionais devem ser examinados com vistas à prevenção do contágio. No entanto, parece-nos mais importante enfatizar outras questões, em geral menosprezadas. No contexto de deterioração dos serviços de saúde públicos, a adequação dos ambientes de trabalho é urgente. Ela deve comportar tanto medidas efetivas de engenharia quanto de organização do trabalho que visem ao controle da exposição ao $M$. tuberculosis no ambiente de trabalho. Penteado (1999) examinou criticamente esses problemas em hospital universitário usando a análise ergonômica como método. Seus achados apontam claramente para a inadequação do ambiente e da organização do trabalho existentes. Entre outros fatores, ocorrem: desproporção entre a área física e o número de leitos; falta de definição de fluxo de ventilação/exaustão nas dependências do serviço; falta de delimitação e adequação do dimensionamento de espaços internos da enfermaria para realização de procedimentos específicos; funcionamento precário do sistema de exaustão e inexistência de filtro HEPA.

Em nosso trabalho, os resultados revelam risco aumentado de adoecer por TB, em relação à população geral, apenas nos grupos etários mais jovens. Esse fato não contradiz, mas antes, reforça o amplo reconhecimento do papel determinante que a exposição ocupacional cumpre nesse adoecimento. Um viés poderia ter ocorrido ao não se computar os profissionais da cidade inscritos em outros conselhos estaduais de enfermagem (essa situação é possível nos casos de processos de transferência de registro em tramitação). Assim, as estimativas para o coletivo de enfermagem que atua nesse município poderiam atenuar a real magnitude do risco desse grupo. Dados da literatura dão suporte ao referido nexo epidemiológico em vários países, industrializados ou em desenvolvimento. Em países em desenvolvimento mostraram-se riscos maiores de adquirir tuberculose-doença em trabalhadores de enfermagem do que na população em geral (TAKEDA; ROBAZZI; LAVRADOR, 2001; KILINC et al., 2002). O risco de tuberculose-infecção também foi investigado, chegando-se a resultados semelhantes (BETANCOUR et al., 2003). No Brasil, considerando a alta morbidade na comunidade e principalmente o

${ }^{5}$ Relatório Anual de Informações Sociais.

Rev. bras. Saúde ocup., São Paulo, 33 (1 17): 06-14, 2008 
processo de pauperização que atinge parcela desses trabalhadores, a hipótese de contato domiciliar é plausível. Tal possibilidade não invalida a análise presente, pois mesmo nessa perspectiva sugere-se excesso de risco; coloca-se, ademais, outro papel reservado ao trabalho, dessa vez não como determinante biológico, mas como social. Assim, a ocupação representaria um nível diferente e mais complexo de determinação do que aqueles que ocupam as variáveis biológicas, tais como referidas em modelos mais reducionistas.

Uma vantagem da abordagem apresentada diz respeito às limitações em se trabalhar com as medidas de exposição (risco-infecção), por envolverem muitos fatores que variam de um local para outro. Mesmo em países desenvolvidos, onde há recursos para pesquisas desse tipo, sobrevêm essas dificuldades, uma vez que a complexidade dos fatores envolvidos impede de predizer diretamente a exposição ao $M$. tuberculosis (FIELD, 2001). A pesquisa de preditores individuais implica na oferta de serviços diagnósticos de qualidade, o que não é uma tarefa simples frente a uma população heterogênea, sobretudo quanto ao tipo de vínculo empregatício, se público ou privado. Esses aspectos elucidam a necessidade de se apropriar de métodos e técnicas condizentes com a realidade estudada, para os fins específicos a que se destinam. Nosso propósito, diferentemente daquele de pesquisa etiológica, foi o de estimar uma medida que forneça condição concreta de análise desse risco no setor saúde, a partir de dados já disponíveis. Em um serviço de referência em TB, ou em uma comunidade de alta prevalência da doença, esse indicador equivale a um diagnóstico coletivo direto. Pode ser de grande interesse para o gestor saber o quanto de sua população trabalhadora está em risco de adoecer e o quanto de esforços de controle do agente infeccioso no meio irá requerer esse adoecimento. Pode ser-lhe de menor utilidade saber se determinado indivíduo $\boldsymbol{x}$ ou $\boldsymbol{y}$ adquiriu a doença por via da exposição ocupacional, configurando "nexo" (objeto de preocupação da Seguridade Social e da Medicina do Trabalho). O foco de suas preocupações, no primeiro caso, estará, portanto, deslocado do indivíduo para o grupo e da clínica para a epidemiologia; enquanto no segundo ocorre o inverso (ênfase no indivíduo). Espera-se que a medida aqui apresentada ampare técnicos e autoridades sanitárias na tomada de suas decisões.

\section{Comentários e perspectivas}

A experiência acumulada em serviços na vigilância e controle da TB facilita a identificação de grupos populacionais em maior risco, dentre os quais os trabalhadores do setor saúde. Esse potencial de uso dos dados epidemiológicos de serviços poderá ser mais bem explorado pela área de Saúde do Trabalhador, mediante a versão Web do Epi-TB, em São
Paulo. É preciso, porém, que se aprimore a qualidade da informação ocupacional coletada no instrumento epidemiológico, para que esta possa balizar não só a investigação do caso, mas também a dos ambientes de trabalho. Para tanto, ela deve incorporar pelo menos dois campos: um para identificar a empresa empregadora e o outro para o local de trabalho, onde se registraria o endereço / bairro (o ideal seria inserir o número de campos necessário para se cobrir os múltiplos vínculos de trabalho existentes nesse setor). Assim, obter-se-iam informações para ações integradas de vigilância em saúde desde o nível local (Unidades de Vigilância em Saúde) em suas interfaces com as Comissões de Controle de Infecção Hospitalar (CCIH's) e com os Serviços Especializados em Engenharia de Segurança e Medicina do Trabalho (SEESMT's). Esse movimento exige a descentralização e horizontalização das ações de vigilância e aderência dos atores envolvidos ao diálogo interinstitucional mediante uma lógica de trabalho complementar e integradora que prevaleça sobre visões estanques e fragmentadoras. Esforços dessa natureza devem ser empreendidos sistematicamente e sob fiança de um compromisso ético com a resolutividade das ações sanitárias.

Os EAS devem ser espaços seguros para o coletivo de trabalhadores e usuários e não apenas locais de cuidados diagnósticos e terapêuticos dirigidos a indivíduos doentes. Nesse sentido, a Vigilância em Saúde do Trabalhador também deve exercer um papel educativo, sensibilizando e capacitando os atores para as melhores práticas institucionais possíveis na proteção da saúde de seus trabalhadores. Acrescente-se que a edição de normas e protocolos técnicos, em caráter complementar aos já existentes, é uma das prerrogativas estratégicas do SUS no município de São Paulo, como atribuição conferida pelo Código Sanitário Municipal.

Como setor regulado pela Vigilância em Saúde, é preciso reconhecer dificuldades de natureza política no setor saúde, quanto à implementação de uma proposta interinstitucional de controle e prevenção. Estas se relacionam aos aspectos legais de competências, ao corporativismo, às assimetrias institucionais e aos conflitos de interesse. Há registro de problemas semelhantes em outros países: nos EUA, por exemplo, os governos estaduais possuem diferentes graus de autoridade reguladora sobre os EAS, complicando a divisão de responsabilidades técnicas e as políticas sanitárias (CENTER FOR DISEASE CONTROL AND PREVENTION, 2005).

Concluímos que a experiência descrita pode ser reproduzida com baixo nível de complexidade e custo em um município como o de São Paulo, onde a vigilância em saúde possui uma razoável estrutura e onde o contingente populacional de trabalhadores em saúde justifica ações de maior envergadura. Reunindo as evidências do levantamento exploratório de 2004 referido anteriormente, as da literatura especializada e as do presente trabalho, algumas indicações 
podem ser sinalizadas. A primeira, de que o estudopiloto aqui apresentado também se presta a um exercício de articulação intrasetorial, esta última sempre interessante à saúde pública, mas que por vezes mostra-se insuficiente ou fugaz. A segunda, de que julgamos ser de importância crucial a análise sistemática dos dados de TB em trabalhadores de saúde para se obter uma série histórica relevante e um quadro mais completo e confiável dessa morbidade. Por fim, acorde o mesmo referencial, de que a incidência de TB em profissionais de enfermagem, pela magnitude de taxas e números absolutos, quando comparados aos de outras categorias de saúde, permite assumir esse grupo como categoria-índice da TB nosocomial. Ainda que refinamentos nos desenhos de estudo possam ser necessários para análises mais acuradas, a exeqüibilidade deste já aponta para a utilidade da abordagem, no monitoramento da TB ocupacional. Em tempos de NR-32, o uso de ferramenta epidemiológica pode e certamente deverá subsidiar a formulação de estratégias de enfrentamento da morbidade do trabalhador de saúde. E, analogamente, de medidas de gestão de risco em EAS.

\section{Agradecimentos}

Ao Centro de Processamento de Dados do Coren, pela colaboração;

Ao grupo técnico CCD-PCT / Covisa, em particular, Dr ${ }^{a}$ Sumie Matai Figueiredo, pela cessão dos dados do EPITB municipal e esclarecimentos pertinentes;

À Rossana Véronica Mendoza López (FSP-USP), pela revisão estatística;

Às Dr ${ }^{a s}$ Laedi Alves Rodrigues dos Santos e Vera Maria Neder Galesi (CVE-SP), pelo encorajamento da proposta e pelas sugestões apresentadas;

Aos colegas da Vigilância em Saúde do Trabalhador / Covisa, especialmente Dr. Luiz Martins Júnior e Dr. José Luis Andrade-Silva, pelos comentários críticos e sugestões;

À Dra Magda Andreotti (Vigilância em Saúde do Trabalhador / Covisa), pelo incentivo permanente e pelos apoios institucional e pessoal.

\section{Referências}

BAGATIIN, E.; ANTÃO, V. C. S.; PINHEIRO, G. A. Vigilância epidemiológica e doenças ocupacionais respiratórias. J. bras. pneumol., Brasília, v. 32, p. S1S4, maio 2006. Suplemento 2.

BETANCOUR, C. A. et al. Riesgo de infección en el trabajador de la salud - Medellín 2001-2002. Acta Méd. Colombiana, Bogotá, v. 28, n. 3, p. 108-111, 2003.

BRASIL. Ministério da Saúde; ORGANIZAÇÃO PAN-AMERICANA DA SAÚDE. Doenças infecciosas e parasitárias relacionadas ao trabalho. In: Doenças relacionadas ao trabalho: manual de procedimentos para os serviços de saúde. Brasília: Ministério da Saúde, 2001. p. 59-64.

BRASIL. Portaria MTE no 485 de 11 de novembro de 2005. Norma regulamentadora segurança e saúde no trabalho em serviços de saúde - NR 32. Diário Oficial da União. Brasília, DF, 16 nov. 2005. Seção 1. Disponível em: http://sbbq.iq.usp.br/arquivos/ seguranca/portaria485.pdf. Acesso em: 15 jul. 2007.

BUVINIC, M.; GIUFFRIDA, A. A; GLASSMAN, A. Gender inequality in work, health, and income. In: HEYMANN, J. Global inequalities at work. New York: Oxford University Press, 2003. p. 200-202.

CENTER FOR DISEASE CONTROL AND

PREVENTION. Guidelines for the investigation of contacts of persons with infectious tuberculosis. United States: MMWR, v. 54, n. RR-15, p. 49-55, 2005.

DEDECCA, C. S. et al. Dimensão ocupacional do setor de atendimento à saúde no Brasil. In: ENCONTRO NACIONAL DE ESTUDOS POPULACIONAIS, 14., 2004, Minas Gerais. Anais eletrônicos... Minas Gerais: Associação Brasileira de Estudos Populacionais, 2004. Disponível em: http://www.abep.org.br/usuario/GerenciaNavegacao. php?caderno_id=433\&nivel=3. Acesso em: 15 jul. 2007.

FIELD, M. J. (Ed.). Tuberculosis in the workplace. Washington, D.C.: National Academy Press, 2001. Disponível em: http://www.nap.edu/catalog/10045. html. Acesso em: 15 jul. 2007.

FRANCO, C.; ZANETTA, D. M. T. Tuberculose em profissionais de saúde: medidas institucionais de prevenção e controle. Arq. Ciênc. Saúde, v. 11, n. 4, p. 244-252, out./dez. 2004.

GIRARDI, S. N.; CARVALHO, C. L. Configurações do mercado de trabalho dos assalariados em saúde no Brasil. Brasília: Organização Pan-Americana de Saúde, 2003. Disponível em: http://www.opas.org. br/rh/areas_det.cfm?id_doc $=163 \& i d \_$area $=4$. Acesso em: 15 jul. 2007. 
KILINC, O. et al. Risk of tuberculosis among healthcare workers: can tuberculosis be considered as an occupational disease? Respir. Med., England, v. 96, n. 7, p. 506-510, july 2002.

KRÜÜNER, A. et al. Tuberculosis as an occupational hazard for health care workers in Estonia. Int. J. Tuberc. Lung. Dis., Paris, v. 5, n. 2, p. 170-176, feb. 2001.

LORENZI, R. L.; ANDRADE-SILVA, J. L. Avaliação da exposição ocupacional à tuberculose em profissionais de saúde no Município de São Paulo, em 2004. Saúde e Soc., São Paulo, v. 14, supl. 1, p. 276-277, 2005.

PENTEADO, E. V. B. F. Tuberculose no ambiente hospitalar: uma questão da saúde do trabalhador.
1999. 135 f. Dissertação (Mestrado em Ciências na área de Saúde Pública) - Escola Nacional de Saúde Pública, Rio de Janeiro, 1999. Disponível em: http://portalteses.cict.fiocruz.br/pdf/FIOCRUZ/1999/ penteadevbfm/capa.pdf. Acesso em: 15 jul. 2007.

RAITIO, M.; HELENIUS, H.; TALA, E. Is the risk of the occupational tuberculosis higher for young health care workers? Int. J. Tuberc. Lung Dis., v. 7, n. 6, p. 556-562, june 2003.

TAKEDA, E.; ROBAZZI, M. L. C. C.; LAVRADOR, M. A. S. Risco ocupacional de adquirir tuberculose entre trabalhadores de enfermagem hospitalar. Rev. bras. Enferm., Brasilia, v. 54, n. 3, p. 456-465, 2001. 